\title{
(-)-DEPRENYL INHIBITS VASCULAR HYPERPERMEABILITY FOLLOWING HEMORRHAGIC SHOCK
}

\author{
Binu Tharakan J., Greg Whaley, Felicia A. Hunter, W. Roy Smythe, and Ed W. Childs \\ Department of Surgery, Texas A\&M Health Science Center College of Medicine and Scott \& White \\ Memorial Hospital, Temple, Texas, USA
}

\begin{abstract}
Recent studies from our laboratory demonstrated the involvement of endothelial cell reactive oxygen species (ROS) formation and activation of apoptotic signaling in vascular hyperpermeability following hemorrhagic shock (HS). The objective of this study was to determine if (-)-deprenyl, an antioxidant with anti-apoptotic properties would attenuate HS-induced vascular hyperpermeability. In rats, HS was induced by withdrawing blood to reduce the MAP to $40 \mathrm{mmHg}$ for 60 minutes followed by resuscitation for 60 minutes. To study hyperpermeability, the rats were injected with FITC-albumin $(50 \mathrm{mg} / \mathrm{kg})$ and the changes in integrated optical intensity of the mesenteric postcapillary venules were obtained intra and extra vascularly utilizing intravital microscopy. Mitochondrial ROS formation and mitochondrial transmembrane potential $(\Delta \Psi \mathrm{m})$ were studied using dihydrorhodamine 123 and JC-1 respectively. Mitochondrial release of cytochrome $c$ was determined using ELISA and caspase-3 activity by a fluorometric assay. Parallel studies were performed in rat lung microvascular endothelial cells (RLMEC) utilizing pro-apoptotic BAK as inducer of hyperpermeability. Hemorrhagic shock induced vascular hyperpermeability, mitochondrial ROS formation, decrease in $\Delta \Psi \mathrm{m}$, release of cytochrome $c$ and caspase- 3 activation $(p<0.05)$. (-)-Deprenyl $(0.15 \mathrm{mg} / \mathrm{Kg})$ attenuated all these effects $(p<0.05)$. Similarly in RLMEC, (-)-deprenyl attenuated BAK peptide induced monolayer hyperpermeability $(p<0.05)$, ROS formation, decrease in $\Delta \Psi \mathrm{m}$, cytochrome $c$ release $(p<0.05)$ and activation of caspase-3 $(p<0.05)$. The protective effects of (-)-deprenyl on vascular barrier functions may be due to its protective effects on $\Delta \Psi \mathrm{m}$ thereby preventing mitochondrial release of cytochrome $c$ and caspase-3 mediated disruption of endothelial adherens junctions.
\end{abstract}

\section{Keywords}

(-)-Deprenyl; ischemia-reperfusion; microvascular permeability; apoptotic signaling; caspase-3

\section{INTRODUCTION}

A recognized important component of the pathophysiology of hemorrhagic shock (HS) is an increase in vascular permeability. This hyperpermeability is secondary to disruption of the microvascular endothelial cell barrier (1-4). The mechanisms underlying the loss of endothelial cell integrity are not fully understood; however, several laboratories, including our own, have shown that an increase in the mediators of apoptosis is present following HS and that endothelial cell apoptosis is associated with vascular hyperpermeability $(1,5-8)$.

Corresponding Author: Ed W Childs, MD Department of Surgery Texas A\&M University Health Science Center College of Medicine \& Scott \& White Memorial Hospital 2401 South 31st Street Temple, Texas, 76508 USA Phone: 254724 4976; Fax: 2547247912 echilds@swmail.sw.org. 
Apoptosis is initiated either via the extrinsic "death ligand" pathway or the intrinsic "mitochondrial" pathway $(5,9)$. The intrinsic apoptotic pathway is mediated by cytochrome $c$, smac, and AIF, all of which are regulated by the balance of proapoptotic (BAK, BAX) and antiapoptotic (Bcl-2, Bcl-xL) proteins belonging to the Bcl-2 family $(5,10-16)$. Initiation of the intrinsic apoptotic pathway begins with release of cytochrome $c$ from the mitochondria. The translocation of cytochrome $c$ in to the cytosol occurs via the mitochondrial transition pores (MTP), which are located in the inner mitochondrial membrane and are high conductance calcium sensitive channels that allow nonselective diffusion. It is the loss of mitochondrial membrane integrity that is responsible for the movement of cytochrome $c$ to the cytosol and the subsequent propogation of the intrinsic apoptotic pathway. Cytochrome $c$, once in the cytosol, acts to release the apoptosome assembly from the apoptotic protease-activating factor-1 (Apaf-1), ATP, and procaspase-9, leading to cellular morphologic and functional alterations via the activation of caspase-3 and caspase-7 (12). We hypothesized that cell-cell detachment during apoptosis may occur as caspase-3 cleaves beta-catenin, an important regulator of the cadherin-mediated cell-cell adhesion in endothelial cells. This catenin-cadherin interaction has been shown to be the most important determinant of junctional strength and paracellular permeability (16-18), and loss of integrity of this interaction leads to significant changes in vascular permeability $(16,19,20)$. We have previously shown that following HS the mitochondrial membrane integrity is compromised, cytosolic cytochrome $c$ levels are increased and caspase- 3 is activated (16).

ROS are generated in all aerobic cells as the result of normal cellular processes, including cytosolic enzymatic reactions as well as the process of oxidative phosphorylation along the mitochondrial electron transport chain during cellular respiration. ROS formation is increased in ischemic states and following HS $(21,22)$. It has previously been shown that ROS can alter MTP and induce the release of cytochrome $c$ from the mitochondria, thereby activating the intrinsic apoptotic pathway (10,23-25). The precise mechanism for modulation of the MTP by ROS is not clear; however, two sites along the membrane have been implicated. The " $\mathrm{S}$ " site is a membrane dithiol whose oxidative status is dependent on ROS. The "P" site remains chemically undefined and its mechanism of action unknown (26-28).

(-)-Deprenyl is a monoamine oxidase-B (MAO-B) inhibitor that has previously been used in the treatment of Parkinson's disease $(29,30)$. The enzyme monoamine oxidase metabolizes biogenic amines such as dopamine and serotonin and produces hydrogen peroxide as a byproduct (29). Hydrogen peroxide is the major source of cellular ROS. Therefore, by inhibiting this enzyme's actions, (-)-deprenyl not only potentiates the effect of dopamine (initially thought to be the main source of benefit in clinical use), but it also decreases ROS levels. Furthermore, (-)-deprenyl has also been shown to have antiapoptotic activity (31-34). Although not fully understood, (-)-deprenyl protects the mitochondria $(32,35)$ and exerts its antiapoptotic effects via alterations in gene expression (ie. (-)-deprenyl increases levels of Bcl-2 and Bcl-xL and decreases levels of BAX $(26,36)$, decreasing oxidative stress (37), and interfering with the action of effector caspases such as caspase-3 (38). Subsequent studies have elucidated the antiapoptotic effect of (-)-deprenyl in other cells including liver and kidney cells $(26,28)$. We postulated that (-)-deprenyl would decrease the production of ROS, inhibit the intrinsic apoptotic pathway, and stabilize the MTP in microvascular endothelial cells following HS.

\section{MATERIALS AND METHODS}

Animals

Male Sprague-Dawley rats (275-325 g) were acquired from Charles River Laboratories (Wilmington, MA), and housed in the animal facility at the Texas A\&M University Health Science Center College of Medicine at Scott \& White Memorial Hospital, Temple, Texas, 
USA. This animal facility is approved by the Association for Assessment and Accreditation of Laboratory Animal Care International, in compliance with the National Institutes of Health guidelines. Approval for all experiments was granted from the Institutional Animal Care and Use Committee. A 12:12 hour dark/light cycle was maintained while housing the rats, and they were given ad libitum access to food and water. The room temperature and humidity in the animal housing were kept at $25^{\circ} \pm 2^{\circ} \mathrm{C}$ and $55 \%$, respectively. The rats were fasted for 18 hours prior to each experiment.

\section{Chemicals and reagents}

(-)-Deprenyl, FITC-albumin and dihydrorhodamine 123 were obtained from Sigma (St. Louis, MO, USA). JC-1 reagent (5,5',6,6' tetrachoro-1,1',3,3' tetraethylbenzimidazolyl carbocyanine iodide) was obtained from Cell Technology Inc. (Mountain View, CA, USA) and prepared by reconstitution of the lyophilized reagent with 500uL of DMSO to yield a 100X stock solution. This 100X solution was diluted 1:100 immediately prior to the experiments in $1 \mathrm{X}$ assay buffer. BAK (BH3) peptide was obtained from R\&D Systems (Minneapolis, MN, USA). TransITLT1 polyamine was obtained from Mirus Bio Corporation (Madison, WI, USA). Cytochrome $c$ ELISA kit was obtained from R\&D Systems (Minneapolis, MN, USA). Caspase-3 assay kit was obtained from Calbiochem (La Jolla, CA). Rat lung microvascular endothelial calls (RLMEC) and the MCDB-3 media were obtained from VEC-technologies (Rensselaer, NY, USA).

\section{Animal surgery and intravital microscopy}

The rats were anesthetized with a single intramuscular injection of $50 \%$ urethane $(1.5 \mathrm{~g} / \mathrm{kg})$. Polyethylene tubing (PE-50, $0.58 \mathrm{~mm}$ ID) was used to cannulate the right internal jugular vein for intravenous administration of normal saline $(3 \mathrm{ml} / \mathrm{hr})$ and to cannulate the right carotid artery for blood withdrawal. The mean arterial pressure was monitored continuously via a PE-50 cannula placed in the left femoral artery and connected to a blood pressure monitor (DigMed, BPA 400A, Micromed, Louisville, KY, USA). A midline laparotomy incision was performed and a section of mesentery from the proximal ileum was exteriorized for examination and draped over a Plexiglas stage. The rats were placed on a temperaturecontrolled Plexiglas platform mounted to an intravital upright microscope (Nikon E600, Tokyo, Japan). Normal saline was superfused over the mesentery at $2 \mathrm{ml} / \mathrm{min}$ and the mesentery was covered with plastic wrap to reduce evaporation. The intravital microscope was used to examine venules with diameters of 20 to $30 \mu \mathrm{m}$ with the Nikon 20x objective, 0.45 to $2.16 \mathrm{~mm}$ working distance (Nikon Instruments, Inc., Natick, MA, USA). Images obtained with a Photometric Cascade Camera (Roper Scientific, Tucson, AZ, USA) were projected onto a computer monitor, and captured digitally on computer disc. The analysis of the data was performed using MetaMorph 4.5/4.6 (Universal Imaging Corp., Downington, PA, USA).

\section{In Vivo Studies}

Experimental protocols-Each experimental group consisted of 5 rats. All rats were allowed 30 minutes following surgical manipulation for recovery. The experimental group of animals then received (-)-deprenyl $(0.15 \mathrm{mg} / \mathrm{kg})$ intravenously and the sham animals received normal saline. After 10 minutes (Shock $\mathrm{T}_{0}$ ), the rats underwent 60 minutes of hemorrhagic shock, produced by withdrawing blood from the right carotid artery in order to decrease the MAP to $40 \mathrm{mmHg}$. This requires withdrawal of approximately 50-60\% of the rat's total blood volume. Following the 60 minute time period, the shed blood plus two times the volume of normal saline was reinfused in order to maintain a MAP equal to or greater than $90 \mathrm{mmHg}$ (shock $\mathrm{T}_{60}$ ). Mesenteric postcapillary venules in a transilluminated segment of small intestine were examined to quantitate changes in albumin flux. Parameters were recorded post-shock at 10 minutes intervals for 60 minutes. The following formula was used for the calculation of 
light intensity: $\Delta \mathrm{I}=\mathrm{Ii}-\mathrm{Io} / \mathrm{Ii}$ where $\Delta \mathrm{I}$ is change in light intensity, Ii is light intensity inside the vessel and Io is light intensity outside the vessel. Gray scale values were measured in the postcapillary venules and in the extravascular space adjacent to the predetermined venule using MetaMorph image analysis system as previously described (16).

ROS formation-The rats were injected intravenously with the fluorescent indicator dihydrorhodamine $123(50 \mathrm{mg} / \mathrm{kg})$ and were subjected to intravital microscopy. Each group consisted of 5 rats. The use of dihydrorhodamine 123 for in vivo visualization of ROS formation in rats was previously published from our laboratory (22). The conversion of dihydrorhodamine 123 to a fluorescent product rhodamine 123 that fluoresces red is positively correlated to mitochondrial ROS formation. The changes in fluorescent intensity that indicate ROS formation were evaluated and recorded utilizing automated image analysis.

Mitochondrial tansmembrane potentail-The rat mesenteric vasculature was exposed and superfused with JC-1 reagent (1:100) for 20 minutes prior to administration of (-)-deprenyl $(0.15 \mathrm{mg} / \mathrm{kg})$, to measure changes in mitochondrial transmembrane potential using intravital microscopy. Each group consisted of 5 rats. The rats were placed on a temperature controlled Plexiglas platform mounted to an intravital upright microscope (Nikon E 600, Tokyo, Japan). Hemorrhagic shock was induced as described above. In normal conditions, in healthy cells, the cationic fluorescent indicator JC-1 fluoresces the mitochondria red (Cy3 filter, emission wave length $590 \mathrm{~nm}$ ) and cytoplasm fluoresces green (FITC filter, emission wave length 530 $\mathrm{nm})$. The negative charge established by the mitochondrial membrane potential allows the lipophilic dye, bearing a delocalized positive charge to enter the mitochondrial matrix where it accumulates. When the critical concentration is exceeded, J-aggregates form which fluoresce red (Cy3). In cells undergoing apoptosis, the mitochondrial membrane potential collapses and the JC-1 can no longer accumulate within the mitochondria. In these cells, JC-1 remains in the cytoplasm in a green fluorescent monomeric form. The FITC or Cy3 fluorescent intensities were measured every 10 minutes up to 60 minutes following resuscitation. The images were captured and the Cy3 fluorescent intensity was calculated.

Cytochrome c release-Each group consisted of 5 rats. Hemorrhagic shock was produced as described above. Cytochrome $c$ assay from the cytosolic fraction of the rat mesenteric tissue was performed by ELISA kit (R\&D Systems, Minneapolis, MN, USA). The tissues were weighed and homogenized in a cold preparation buffer. The tissue homogenates were centrifuged $\left(10,000 \times \mathrm{g}\right.$ for $60 \mathrm{~min}$ at $\left.4^{\circ} \mathrm{C}\right)$ and the supernatant (cytosolic fraction) was collected and subjected to protein assay. The samples were treated with a conjugate reagent, transferred to an antibody coated microwell plate and incubated for 60 minutes at room temperature. The wells were washed using a wash solution. The samples were then treated with a peroxidase substrate reagent and incubated for 15 minutes at room temperature. Following the addition of a stop solution, the samples were read at $450 \mathrm{~nm}$ within 30 minutes. A serial dilution of cytochrome $c$ standard was used in the assay along with the samples. The values of cytochrome $c$ standards were plotted on semi-log and the unknown values determined based on the standard plot.

Caspase-3 activity-Each group consisted of 5 rats. Caspase-3 activity was determined using a fluorescent method (Calbiochem, La Jolla, CA) where active caspase-3 cleaves after aspartate residues in a particular peptide sequence (DEVD). The DEVD substrate was labeled with a fluorescent molecule, 7-amino-4-trifluoromethyl coumarin. The mesenteric microvessels harvested from the animals were homogenized in caspase-3 sample lysis buffer provided in the kit. The homogenates were centrifuged at $10000 \mathrm{~g}$ for 20 minutes at $4^{\circ} \mathrm{C}$. The resulting supernatant was used for protein estimation and caspase- 3 assays. The tissue lysates were treated with the substrate conjugate and the resulting fluorescent intensity was measured 
in a fluorescent plate reader at excitation $400 \mathrm{~nm}$ and emission $505 \mathrm{~nm}$. The data are expressed as a percentage of the sham-control values.

\section{In Vitro Studies}

Monolayer permeability-RLMEC were cultured on fibronectin-coated dishes in MCDB-3 media supplemented with $10 \%$ fetal bovine serum. These cells were later grown as monolayers on Costar Transwell membranes for 48 hours. Media was exchanged for phenol red-free media one hour prior to beginning the experiments. TransIT-LT1 was exposed with serum-free media at a concentration of 1:5, and then BAK (BH3) $(5 \mu \mathrm{g} / \mathrm{ml})$ was prepared in TransIT for 15 minutes prior to exposure to the monolayers. Next, the monolayers in the luminal (upper) chamber were exposed to BAK (BH3) peptide for 1 hour, using TransIT-LT1 polyamine. The luminal chamber contained $100 \mu \mathrm{l}$ medium and the abluminal (lower) chamber contained 600 $\mu \mathrm{l}$ medium. One group of monolayers was pretreated with (-)-deprenyl at a concentration of $10 \mu \mathrm{M}$ for 1 hour prior to exposure to BAK. (-)-Deprenyl alone or TransIT alone treated cells served as control groups. Each group consisted of 5 replicates. Finally, FITC-albumin $(5 \mathrm{mg} /$ $\mathrm{ml}$ ) was added to the luminal chamber of the Transwell plates and incubated at $37^{\circ} \mathrm{C}$ for 30 minutes, the fluorescent intensity of samples taken from the abluminal chambers was measured using a fluorometric plate reader at excitation $494 \mathrm{~nm}$ and $520 \mathrm{~nm}$. These values were calculated as a percentage of the control values.

ROS formation-RLMEC were grown on fibronectin coated chamber slides for 24 hours. The cells were exposed to media without phenol red for 1 hour and were treated for 1 hour with (-)-deprenyl $(10 \mu \mathrm{M})$. BAK (BH3) peptide $(5 \mu \mathrm{g} / \mathrm{ml})$ was exposed to the transfection medium (TransIT) for 15 minutes and the transfection was performed for 1 hour. Untreated (basal) cells served as controls during various experiments. Each group consisted of 5 replicates. The cells were treated with dihydrorhodamine 123 (Invitrogen; $5 \mu \mathrm{M}$ ) for 30 minutes. The cells were repeatedly washed in PBS and observed under a fluorescent microscope.

Mitochondrial Membrane Potential-To determine the mitochondrial membrane potential, RLMEC were grown on fibronectin coated chamber slides for 24 hours and exposed to media without phenol red for 1 hour. BAK (BH3) peptide $(5 \mu \mathrm{g} / \mathrm{ml})$ was exposed to the transfection medium (TransIT-LT1 polyamine) for 15 minutes prior to transfection for 1 hour, as described above. (-)-Deprenyl $(10 \mu \mathrm{M})$ was exposed to the cells 1 hour prior to BAK (BH3) transfection. Untreated and (-)-deprenyl alone treated cells served as controls. Each group consisted of 5 replicates. The cells were incubated with JC-1 (5, $5^{\prime}, 6,6^{\prime}$ 'tetrachoro- $1,1^{\prime}, 3,3^{\prime}$ ' tetraethylbenzimidazolyl carbocyanine iodide) for 15 minutes at $37^{\circ} \mathrm{C}$, washed in PBS and observed immediately under a fluorescent microscope.

Cytochrome c release-RLMEC were grown on fibronectin-coated culture dishes at $37^{\circ}$ $\mathrm{C}$ with $5 \% \mathrm{CO}_{2}$ as described above. BAK (BH3) peptide $(5 \mu \mathrm{g} / \mathrm{ml})$ was exposed to the transfection medium (TransIT) for 15 minutes and the transfection was performed for 1 hour. (-)-Deprenyl $(10 \mu \mathrm{M})$ was exposed to the cells 1 hour prior to BAK (BH3) transfection. Untreated and (-)-deprenyl alone treated cells served as controls. Each group consisted of 5 replicates. The cells were lysed in a cold preparation buffer provided in the kit, centrifuged $\left(10,000 \times \mathrm{g}\right.$ for $60 \mathrm{~min}$ at $\left.4^{\circ} \mathrm{C}\right)$ and the supernatant (cytosolic fraction) was collected and subjected to protein assay. The cytosolic cytochrome $c$ levels were estimated using a cytochrome $c$ ELISA kit as described above.

Caspase-3 activity-RLMEC were cultured in fibronectin-coated dishes and exposed to (-)-deprenyl $(10 \mu \mathrm{M})$ for 1 hour. They were then transfected with BAK (BH3) peptide as described above. (-)-Deprenyl $(10 \mu \mathrm{M})$ was exposed to the cells 1 hour prior to BAK (BH3) 
transfection. Untreated and (-)-deprenyl alone treated cells served as controls. Each group consisted of 5 replicates. The cells were collected in lysis buffer and centrifuged at 10,000 $\times$ g 15 minutes at $4{ }^{\circ} \mathrm{C}$. Following protein estimation, the supernatant was collected and caspase- 3 activity was determined by fluorometric assay as described above.

Statistical analysis-All data are expressed as mean + SE. Groups were compared using analysis of variance (ANOVA) and subsequent bonferroni's post-test for multiple comparisons. Students's t-test was also employed. Experimental values were compared to the initial baseline value and expressed as percentage change. A $p$ value of $<0.05$ was considered a significant difference.

\section{RESULTS}

\section{In Vivo Studies}

(-)-Deprenyl inhibits hemorrhagic shock-induced vascular hyperpermeabilityThe first image in figure 1A is a composite image of a rat's mesenteric post-capillary venule prior to HS demonstrating minimal extravasation of FITC-albumin into the extravascular space. The second image taken from a rat after 60 minutes of $\mathrm{HS}$ and 60 minutes of resuscitation $\left(\mathrm{T}_{60}\right)$, demonstrates extravasation of FITC albumin into the extravascular space. The third image corresponds to (-)-deprenyl pre-treatment followed by hemorrhagic shock for 60 minutes and 60 minutes of resuscitation (shock $\mathrm{T}_{60}$ ). (-)-Deprenyl significantly attenuated the extravasation of FITC albumin into the extravascular space. Figure 1B is a graphical representation of the fluorescent intensity of the extravascular region surrounding the mesenteric vasculature. An increased fluorescent intensity signifies increased vascular permeability due to extravasation of FITC-albumin. HS resulted in a significant increase in vascular permeability $(p<0.05)$. Pretreatment with (-)-deprenyl significantly inhibited this effect $(p<0.05)$. Fluourescent intensity in the (-)-deprenyl pretreatment group was not significantly different than the sham group.

(-)-Deprenyl inhibits hemorrhagic shock-induced ROS formation-(-)-Deprenyl $(0.15 \mathrm{mg} / \mathrm{kg})$ treatment 10 minutes prior to the hemorrhagic shock period resulted in significant attenuation of ROS formation compared to the hemorrhagic shock group without (-) (-)deprenyl treatment $(p<0.05$; Figure 2A, 2B). In figure 2A, the first image is a composite image of a rat mesenteric post-capillary venule from pre-shock period demonstrating minimal ROS formation. The second image taken from a rat after 60 minutes of HS and 60 minutes of resuscitation (Shock $\mathrm{T}_{60}$ ) demonstrates increased ROS formation. The third image in figure $2 \mathrm{~A}$ represents attenuation of ROS formation in (-)-deprenyl pre-treatment followed by hemorrhagic shock. Figure 2B is the graphic representation of the changes in ROS formation in sham-control, hemorrhagic shock group and hemorrhagic shock group pre-treated with (-)deprenyl.

\section{(-)-Deprenyl prevents hemorrhagic shock induced mitochondrial}

transmembrane depolarization-In normal conditions, the cationic dye JC-1exists as monomer, which fluoresces green in the cytosol and also accumulates as J-aggregates in the mitochondria, which fluoresce as red. Upon activation of apoptotic signaling, JC-1 exists only in a monomeric form (green). Figure 3A shows the image of a mesenteric post-capillary venule from a sham-control rat demonstrating both green (cytosol) and red (mitochondria) fluorescence in the vascular endothelial cells. Following shock $\left(\mathrm{T}_{60}\right)$ there was a decrease in red (mitochondrial) fluorescence indicating the loss of mitochondrial transmembrane potential. (-)-Deprenyl $(0.15 \mathrm{mg} / \mathrm{kg})$ pretreatment prevented the loss of mitochondrial transmembrane potential evidenced by the increase in red fluorescence compared to the hemorrhagic shock 
group without (-)-deprenyl treatment (Figures 3A, 3B). Figure 3B is the graphic representation of the changes in the fluorescence of J-aggregates (red; Cy3 filter).

(-)-Deprenyl inhibits hemorrhagic shock-induced cytochrome c release-The depolarization of mitochondrial membrane is known to result in the mitochondrial release of cytochrome $c$ to the cytosol. The cytochrome $c$ levels from each experimental group are depicted in figure $4 \mathrm{~A}$. Tissue obtained at Shock $0\left(\right.$ Shock $\left.\mathrm{T}_{0}\right)$ and sixty minutes post-shock (Shock $\mathrm{T}_{60}$ ) had a significantly higher cytochrome $c$ level when compared to the control (Sham) group $(p<0.05)$. This effect was inhibited by pretreatment with (-)-deprenyl $(p<0.05)$.

(-)-Deprenyl inhibits hemorrhagic shock-induced caspase-3 activation-The hemorrhagic shock group (Shock $\mathrm{T}_{0}$ and $\mathrm{T}_{60}$ ) showed significant increase in caspase- 3 activity compared to sham-control group ( $p<0.05$; Figure 4B). The hemorrhagic shock group $\left(\mathrm{T}_{60}\right)$ that was treated with (-)-deprenyl prior to shock showed significantly low caspase-3 activity compared to hemorrhagic shock group $\left(\mathrm{T}_{60}\right)$ without (-)-deprenyl pre-treatment $(p<0.05$; Figure 4B).

\section{In vitro Studies}

(-)-Deprenyl inhibits BAK-induced hyperpermeability in RLMEC-Figure 5A reflects the monolayer permeability of different experimental groups as a percentage of the TransIT-control group. Measurement of the fluorescent intensity of the media from the abluminal chamber revealed that BAK transfection significantly increased monolayer permeability when compared to TransIT-controls $(p<0.05)$. Pretreatment with (-)-deprenyl inhibited the increase in permeability that BAK induced $(p<0.05)$.

(-)-Deprenyl inhibits BAK-induced ROS formation in RLMEC-In RLMEC, transfection of BAK (BH3) peptide $(5 \mu \mathrm{g} / \mathrm{ml})$ resulted in increased ROS formation as evidenced by the increase in red fluorescence (Figure 5B). The fluorescence was intense in cells transfected with BAK (BH3) peptide in comparison to untreated control cells or (-)-deprenyl alone treated cells. Pre-treatment of the cells with (-)-deprenyl resulted in a decrease in fluorescence in BAK (BH3) transfected cells indicating a decrease in ROS formation.

\section{(-)-Deprenyl inhibits BAK-induced mitochondrial membrane potential collapse} in RLMEC-In normal cells the cationic fluorescent dye JC-1, fluoresces red within the mitochondria and green in the cytoplasm. In apoptotic cells, with loss of integrity of the mitochondrial membrane, the dye does not accumulate within the mitochondria. As a result, the reagent remains in the cytosol and there is a decrease in red fluorescence. Figure 6A includes images from each of the experimental groups. BAK transfection resulted in decreased red fluorescence compared to control cells and (-) deprenyl pretreatment inhibited this effect.

(-)-Deprenyl prevents BAK-induced cytochrome c release in RLMEC-In BAK (BH3) transfected cells, cytosolic levels of cytochrome $c$ were significantly higher compared to the control group ( $p<0.05$; Figure 6B). Cytoplasmic cytochrome $c$ levels were significantly decreased in BAK (BH3)-transfected cells pre-treated with (-)-deprenyl in comparison to BAK (BH3)-transfected cells without (-)-deprenyl pre-treatment ( $p<0.05$; Figure-6B).

\section{(-)-Deprenyl inhibits BAK-induced caspase-3 activation in RLMEC-In BAK}

(BH3) transfected cells, caspase-3 activity was significantly high compared to the control group $(p<0.05$; Figure 6C). The caspase-3 activity was significantly decreased in BAK (BH3)transfected cells pre-treated with (-)-deprenyl in comparison to BAK (BH3)-transfected cells without (-)-deprenyl pre-treatment $(p<0.05$; Figure 6C). 


\section{DISCUSSION}

In this study, we have shown that HS induces an increase in vascular permeability and that this increase may be due to activation of the intrinsic apoptotic pathway in microvascular endothelial cells. Furthermore, we have shown that (-)-deprenyl effectively prevents this sequence of events by decreasing mitochondrial ROS formation, stabilizing the mitochondrial membrane potential, preventing the release of cytochrome $c$ into the cytosol, and decreasing caspase-3 activity.

The antiapoptotic effects of (-)-deprenyl have been demonstrated in a variety of tissues and pathologic conditions. Treatment with much lower concentrations of (-)-deprenyl produces these antiapoptotic effects than are required for monoamine oxidase (MAO) inhibition (26, $36,39,40)$. Thus, this suggests (-)-deprenyl has an additional mechanism(s) of action for apoptosis inhibition $(41,42)$. One possible mechanism is modulation of gene expression and alteration of protein levels of members of the Bcl-2 protein family. As previously mentioned, the ratio of death promoters (BAX, BAK) to death inhibitors (Bxl-2, Bcl-xL) controls programmed cell death $(5,10-16)$. Proapoptotic members of this protein family accomplish cell death by increasing ROS generation, releasing apoptogenic factors, directly or indirectly affecting the state of MTP, and activating executors of apoptosis (caspases, DNAses) (13). Antiapoptotic members of the Bcl-2 family, however, act by counter-regulatory mechanisms, either sequestering proteases or interfering with the release of apoptosis-promoting factors (13). (-)-Deprenyl has previously been shown to increase levels of Bcl-2 and Bcl-xL and to decrease levels of BAX $(31,32,39)$.

A second possible mechanism of action for (-)-deprenyl is modulation of the levels of antioxidant proteins. It has been shown that (-)-deprenyl decreases hydroxyl radical levels and increases levels of the important free radical scavenger superoxide dismutase 1 and 2 (29). ROS levels are a significant determinant of mitochondrial membrane stability (23). (-)Deprenyl may exert its antiapoptotic action by decreasing the levels of free radicals within the affected cells.

Oxidative stress has been implicated in the vascular hyperpermeability associated with ischemia-reperfusion injury (24). The oxygen-derived free radicals that are produced when oxygen is re-introduced into an ischemic system can selectively damage endothelial cells (25). While ROS have been shown to be involved in systemic vascular hyperpermeability, the specific sites and mechanisms of action are not clear. Furthermore, the interplay between ROS and apoptotic signaling pathways is not yet fully known. ROS are generated within the mitochondria as well as by various cytoplasmic enzymes $(43,44)$. However, the mitochondrial respiratory chain has been shown to be the major intracellular source of ROS (45). Specifically, Complexes I (NAPH dehydrogenase) and III (Q-cycle) are the primary sites of ROS generation along the mitochondrial electron transport chain (45).

The link between mitochondrial ROS generation and apoptotic signaling is not yet fully elucidated; however, cytochrome $c$ release has been shown to be regulated, at least in part, by ROS generation (46). Inhibition of the MPT has been demonstrated to prevent the release of cytochrome $c$ into the cytoplasm (23). We have previously demonstrated that the mitochondrial Complex III was involved in pro-apoptotic BAK induced ROS generation, cytochrome $c$ release, and endothelial cell hyperpermeability (10). Furthermore, we have shown that inhibition of Complex III with antimycin A prevents ROS formation and attenuates hyperpermeability (10). Others have also shown that mitochondrial Complex III is required for ROS generation in ischemic or hypoxic states $(10,46,47)$. (-)-Deprenyl may decrease oxidative stress by modulating the amount of ROS within the cell. (-)-Deprenyl may act via directly scavenging free radicals; however, its ability to affect ROS levels is most likely 
primarily dependent on its interaction with other important scavenging molecules (29).

Scavenging of ROS generated within the mitochondria may prevent activation of the intrinsic apoptotic pathway, opening of the MTP, release of cytochrome $c$ into the cytoplasm, and the associated vascular hyperpermeability.

Yet another theory regarding the antiapoptotic activity of (-)-deprenyl is related to its chemical structure. The molecule has a tertiary amino group making it structurally similar to several other molecules that have an effect described as a general inhibitory effect on most $\mathrm{K}+$-channels $(26,48)$. It is postulated that the MTP has similar structural features allowing (-)-deprenyl to bind to the MTP and to directly inhibit it's opening (48).

In conclusion, our results indicate that (-)-deprenyl is an effective inhibitor of vascular hyperpermeability following HS. We have shown that (-)-deprenyl inhibits in vitro ROS generation, loss of mitochondrial membrane potential, and microvascular hyperpermeability as well as in vivo ROS generation, cytochrome c release, and vascular hyperpermeability following HS. Inhibition of the intrinsic apoptotic pathway, either by alteration of gene expression levels of proteins involved in the regulation of programmed cell death, decreased oxidative stress, a direct action of the molecule on the MTP, or a combination of these actions, may be responsible for (-)-deprenyl's effect on vascular permeability following HS. Further study may be undertaken regarding the clinical efficacy of (-)-deprenyl to reduce vascular hyperpermeability in conditions such as HS.

\section{Acknowledgments}

This work was supported by a grant (K01HL07815-01A1), from National Heart, Lung and Blood Institute, National Institutes of Health, USA.

\section{REFERENCES}

1. Tharakan B, Hunter RA, Smythe WR, Childs EW. Alpha-lipoic acid attenuates hemorrhagic shockinduced apoptotic signaling and vascular hyperpermeability. Shock 2008;30(5):571-7. [PubMed: 18923301]

2. Baluk P, Hirata A, Thurston G, Fujiwara T, Neal CR, Michel CC, McDonald DM. Endothelial gaps: time course of formation and closure in inflamed venules of rats. Am J Physiol 1997;272:L155-170. [PubMed: 9038915]

3. Childs EW, Udobi KF, Hunter FA, Dhevan V. Evidence of transcellular albumin transport after hemorrhagic shock. Shock 2005;23:565-570. [PubMed: 15897811]

4. McDonald DM. Endothelial gaps: plasma leakage during inflammation. News Physiol Sci 1998;13:104-105. [PubMed: 11390771]

5. Davidson MT, Deitch EQ, Lu Q, Hasko G, Abungu B, Nemeth ZH, Zaets SB, Gaspers LD, Thomas AP, Xu DZ. Trauma-hemorrhagic shock mesenteric lymph induces endothelial apoptosis that involves both caspase-dependent and caspase-independent mechanisms. Ann Surg 2004;240:123-131. [PubMed: 15213628]

6. Mauriz JL, Gonzalez P, Jorquera F, Olcoz JL, Gonzalez-Gallego J. Caspase inhibition does not protect against liver damage in hemorrhagic shock. Shock 2003;9:33-37. [PubMed: 12558141]

7. Murao Y, Hata M, Ohnishi K, Okuchi K, Nakajima Y, Hiasa Y, Junger WG, Hoyt DB, Ohnishi T. Hypertonic saline resuscitation reduces apoptosis and tissue damage of the small intestine in a mouse model of hemorrhagic shock. Shock 2003;20:23-28. [PubMed: 12813364]

8. Watts JA, Grattan RM, Whitlow BS, Kline JA. Activation of poly(ADP-ribose) polymerase in server hemorrhagic shock and resuscitation. Am J Physiol Gastrointest Liver Physiol 2001;281:G498-506. [PubMed: 11447030]

9. Honda HM, Korge P, Weiss JN. Mitochondria and ischemia/reperfusion injury. Ann NY Acad Sci 2005;1047:248-258. [PubMed: 16093501] 
10. Childs EW, Tharakan B, Hunter FA, Isong M, Liggins ND. Mitochondrial complex III is involved in proapoptotic BAK-induced microvascular endothelial cell hyperpermeability. Shock 2008;29(5): 636-641. [PubMed: 18414238]

11. Gottlieb E, Vander Heiden MG, Thompson CB. Bcl-x(L) prevents the initial decrease in mitochondrial membrane potential and subsequent reactive oxygen species production during tumor necrosis factor alpha-induced apoptosis. Mol Cell Biol 2002;20:5680-5689. [PubMed: 10891504]

12. Kim R, Emi M, Tanabe K. Caspase-dependent and -independent cell death pathways after DNA damage. Oncol Rep 2005;14:595-599. [PubMed: 16077961]

13. Kim R, Emi M, Tanabe K. Role of mitochondrial as the gardens of cell death. Cancer Chemother Pharmacol 2005;57:1-9. [PubMed: 16001171]

14. Kuwana T, Bouchier-Hayes L, Chipuk JE, Bonzon C, Sullivan BA, Green DR, Newmeyer DD. BH3 domains of $\mathrm{BH} 3$-only proteins differentially regulate Bax-mediated mitochondrial membrane permeabilization both directly and indirectly. Mol Cell 2005;17:525-535. [PubMed: 15721256]

15. Narita M, Shimizu U, Ito T, Chittenden T, Lutz RJ, Matsuda H, Tsujimoto Y. Bax interacts with the permeability transition pore to induce permeability transition and cytochrome c release in isolated mitochondrial. Proc Natl Acad Sci USA 1998;95:14681-14686. [PubMed: 9843949]

16. Childs EW, Tharakan B, Hunter FA, Tinsley JH, Cao X. Apoptotic signaling induces hyperpermeability following hemorrhagic shock. Am J Physiol Heart Circ Physiol 2007;292:H3179_ 189. [PubMed: 17307990]

17. Del Maschio A, Zanetti A, Corada M, Rival Y, Ruco L, Lampugnani HC, Dejana E. Polymorphonuclear leukocyte adhesion triggers the disorganization of ehdothelial cell-to-cell adherens junctions. J Cell Biol 1996;35:497-510. [PubMed: 8896605]

18. Wong RK, Baldwin AL, Heimark RL. Cadherin-5 redistribution at sites of TNF-alpha and IFNgamma-induced permeability in mesenteric venules. Am J Physiol 1999;276:736-748.

19. Gumbiner BM, Yamada KM. Cell-to-cell contact and extracellular matrix. Curr Opin Cell Biol 1995;7:615-618. [PubMed: 8573334]

20. Hinck L, Nathke IS, Papkoff J, Nelson WJ. Beta-catenin: a common target for the regulation of cell adhesion by Wnt-1 and Src signaling pathways. Trends Biochem Sci 1994;19:538-542. [PubMed: 7846766]

21. Childs EW, Udobi KF, Hunter FA. Hypothermia reduces microvascular permeability and reactive oxygen species expression after hemorrhagic shock. J Trauma 2005;58:271-277. [PubMed: 15706187]

22. Childs EW, Udobi KF, Wood JG, Hunter FA, Smalley DM, Cheung LY. In vivo visualization of reactive oxidants and leukocyte-endothelial adherence following hemorrhagic shock. Shock 2002;18:423-427. [PubMed: 12412620]

23. Chen Q, Chai YC, Mazumber S, Jiang C, macklis RM, Chisolm GM, Almasan A. The late increase in intracellular free radical oxygen species during apoptosis is associated with cytochrome c release, caspase activation, and mitochondrial dysfunction. Cell Death Differ 2003;10:323-334. [PubMed: 12700632]

24. Zhao K, Zhao GM, Wu D, Soong Y, Birk AV, Schiller PW, Szeto HH. Cell-permeable peptide antioxidants targeted to inner mitochondrial membrane inhibit mitochondrial swelling, oxidative cell death, and reperfusion injury. J Biol Chem 2004;279:34682-34690. [PubMed: 15178689]

25. Ricci JE, Gottlieb RA, Green DR. Caspase-mediated loss of mitochondrial function and generation of reactive oxygen species during apoptosis. J Cell Biol 2003;160:65-75. [PubMed: 12515825]

26. De Marchi U, Pietrangeli P, Marcocci L, Mondovi B, Toninello A. L-Deprenyl as an inhibitor of menadione-induced permeability transition in liver mitochondrial. Biochem Pharm 2003;66:17491754. [PubMed: 14563485]

27. Bernardi P. The permeability transition port. Control points of a cyclosporine A-sensitive mitochondrial channel involved in cell death. Biochim Biophys Acta 1996;1275:5-9. [PubMed: 8688451]

28. Costantini P, Chernyak BV, Petronilli V, Bernardi P. Selective inhibition of the mitochondrial permeability transition port at the oxidation-reduction sensitive dithiol by monobromobimane. FEBS Lett 1995;362:239-242. [PubMed: 7536690] 
29. Magyar K, Palfi M, Tabi T, Kalaz H, Szende B, Szoko E. Pharmacological aspects of (-)-deprenyl. Curr Med Chem 2004;11:2017-2031. [PubMed: 15279565]

30. Magyar K, Vizi ES, Ecseri Z, Knoll J. Comparative pharmacological analysis of the optical isomers of phenyl-isopropyl-methyl-propinylamine (E-250). Acta Physio Acad Sci Hung 1967;32:377-387.

31. Toronyi E, Hamar J, Magyar K, Szende B. Antiapoptotic effect of (-)-deprenyl in rat kidney after ischemia-reperfusion. Med Sci Monit 2002;8:BR65-68. [PubMed: 11859269]

32. Wadia JS, Chalmers-Redman ME, Ju WJ, Carlile GW, Phillips JL, Fraser AD, Tatton WG. Mitochondrial membrane potential and nuclear changes in apoptosis caused by serum and nerve growth factor withdrawal: time course and modification by (-)-deprenyl. J Neurosci 1998;18(3):932947. [PubMed: 9437015]

33. Tatton WG, Ju WYL, Holland DP, Tai C, Kwan M. Deprenyl reduces PC12 cell apoptosis by inducing new protein synthesis. J Neurochem 1994;63:1572-1575. [PubMed: 7931312]

34. Magyar K, Szende B, Lengyel J, Tarczali J, Szatmary I. The pharmacology of B-type selective monoamine oxidase inhibitors; milestones in (-) deprenyl research. J Neural Transm 1998;48(Suppl): 29-43.

35. Magyar K, Szenda B, Lengyl J, Tarczali J, Szatmary I. The neuroprotective and neuronal rescue effects of (-) deprenyl. J Neural Transm 1998;52(Suppl):109-123.

36. Xu L, Ma J, Seigel GM, Ma JX. L-Deprenyl blocking apoptosis and regulating gene expression in cultured retinal neurons. Biochem Pharmacol 1999;58:1183-1190. [PubMed: 10484076]

37. Lee CS. Monoamine oxidase inhibitors attenuate cytotoxicity of 1-methyl-4-phenylpyridinium by suppressing mitochondrial permeability transition. Korean J Physiol Pharmacol 2006;10:207-212.

38. Szende B, Bokonyi G, Bocsi J, Keri G, Timar F, Magyar K. Anti-apoptotic and apoptotic action of (-)-deprenyl and its metabolites. J Neural Transm 2001;108:25-33. [PubMed: 11261744]

39. Tatton WG, Chalmers-Redman RM. Modulation of gene expression rather than monoamine oxidase inhibition: (-)-deprenyl-related compounds in controlling neurodegeneration. Neurology 1996;47:S171-183. [PubMed: 8959986]

40. Tatton WG, Chalman-Redman RM, Ju WJ, Mammen M, Carlile GW, Pong AW, Tatton NA. Propargylamines induce antiapoptotic new protein synthesis in serum- and nerve growth factor (NGF)-withdrawn, NGF-differentiated PC-12 cells. J Pharmacol Exp Ther 2002;301:753-764. [PubMed: 11961082]

41. Tatton WG, Ju W, Wadia J, et al. (-)-Deprenyl reduced neuronal apoptosis by maintaining Bcl-2 synthesis and mitochondrial membrane potential. Mov Disord 1994;9:4.

42. Wu RM, Chen RC, Chiueh CC. Effect of MAO-B inhibitors on MPP toxicity in vivo. Ann NY Acad Sci 2000;899:255-261. [PubMed: 10863544]

43. Santore MT, McClintock DS, Lee VY, Budinger GR, Chandel NS. Anoxia-induced apoptosis occurs through a mitochondrial-dependent pathway in lung epithelial cells. Am J Physiol Lung Cell Mol Physiol 2002;282:L727-734. [PubMed: 11880298]

44. Zulueta JJ, Sawhney R, Yu FS, Cote CC, Hassoun PM. Intracellular generation of reactive oxygen species in endothelial cells exposed to anoxia-reoxygenation. Am J Physiol 1997;272:L897-902. [PubMed: 9176254]

45. Turrens JF. Mitochondrial formation of reactive oxygen species. J Physiol (Lond.) 2003;552:335344. [PubMed: 14561818]

46. Guzy RD, Hoyos B, Robin E, Chen H, Liu L, Manfield KD, Simon MC, Hammerling U, Schumacker PT. Mitochondrial complex III is required for hypoxia-induced ROS production and cellular oxygen sensing. Cell Metab 2005;1:1401-1408.

47. Chandel NS, McClintock DS, Feliciano CE, et al. Reactive oxygen species generated at mitochondrial complex III stabilize hypoxia-inducible factor-1alpha during hypoxia: a mechanism of $\mathrm{O}_{2}$ sensing. J Biol Chem 2000;275:25130-25138. [PubMed: 10833514]

48. Halestrap AP, Davidson AM. Inhibition of $\mathrm{Ca}^{2+}$-induced large-amplitude swelling of liver and heart mitochondria by cyclosporine is probably caused by the inhibitor binding to mitochondrial-matrixpeptidyl-prolyl cis-trans isomerase and preventing it interacting with the adenine nucleotide translocase. Biochem J 1990;268:153-160. [PubMed: 2160810] 
A

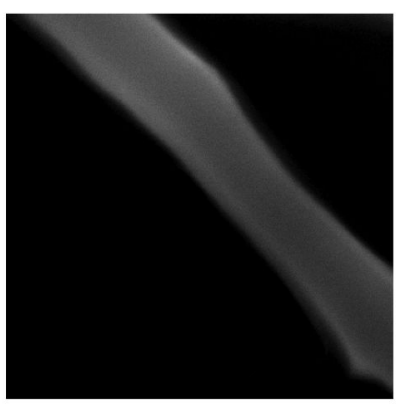

Pre-shock

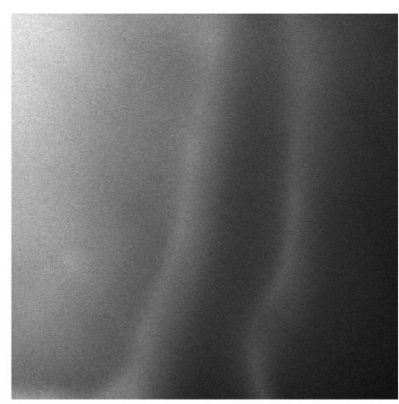

Shock $\mathbf{T}_{60}$

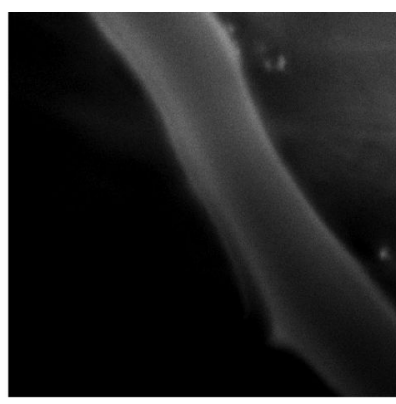

(-)-Deprenyl + Shock T $_{60}$

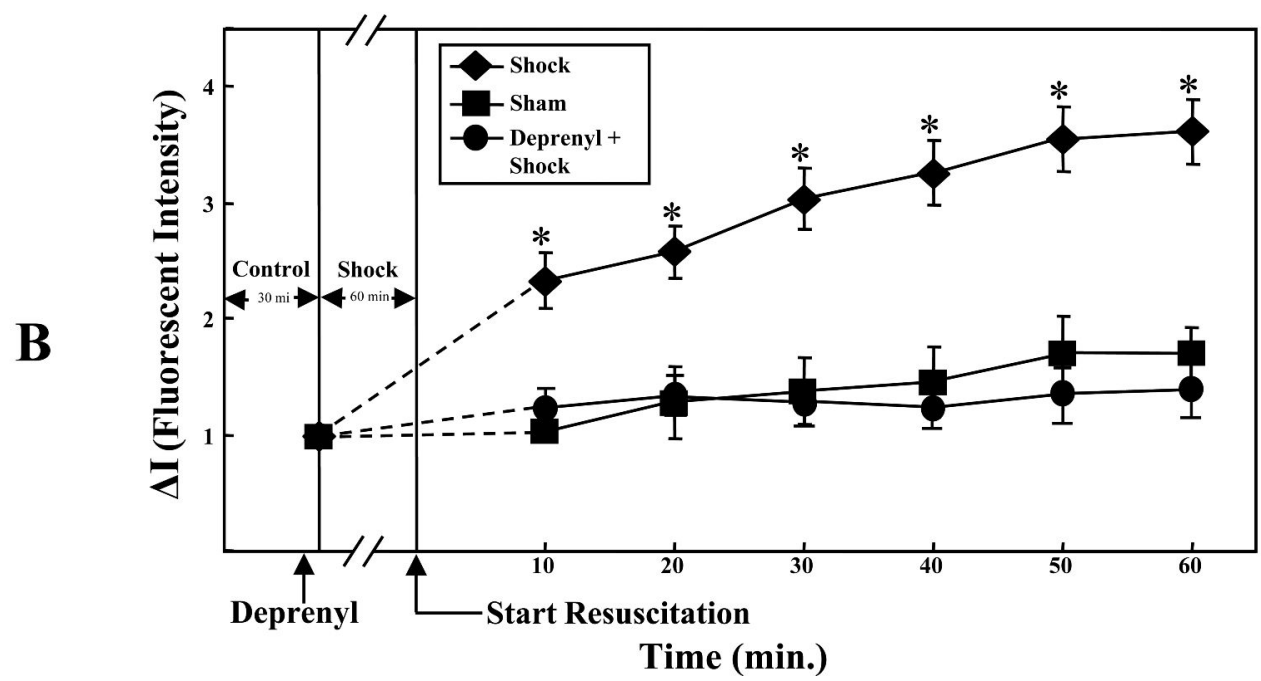

Figure 1.

A. (-)-Deprenyl decreases hemorrhagic shock induced vascular hyperpermeability in rat mesentery post-capillary venules. The images of mesenteric post-capillary venules of preshock, hemorrhagic shock for 1 hour followed by 60 minutes of resuscitation (Shock $\mathrm{T}_{60}$ ) and (-)-deprenyl treatment 10 minutes prior to hemorrhagic shock are shown. FITC-albumin extravasation into the extravascular space is observed following hemorrhagic shock where as (-)-deprenyl treatment prevented the extravasation. B. Vascular permeability is expressed as change in fluorescent intensity inside the vessel compared to the intensity outside the vessel. Hemorrhagic shock induces vascular hyperpermeability (FITC-albumin extravasation) compared with sham-control group. (-)-Deprenyl treatment prevents HS-induced hyperpermeability compared with hemorrhagic shock group without (-)-deprenyl treatment. * $(p<0.05 ; \mathrm{n}=5)$. 


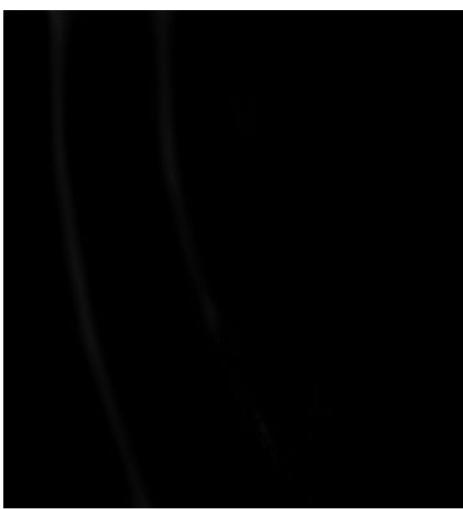

Pre Shock

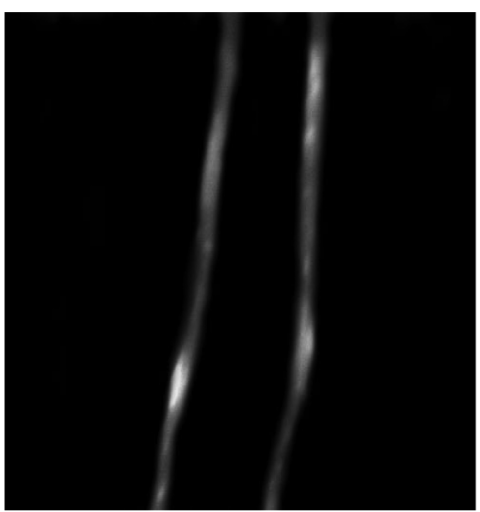

Shock $\mathbf{T}_{60}$

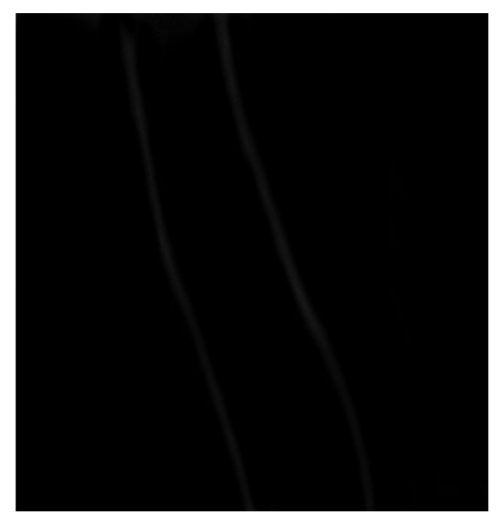

(-)-Deprenyl + Shock $\mathbf{T}_{60}$

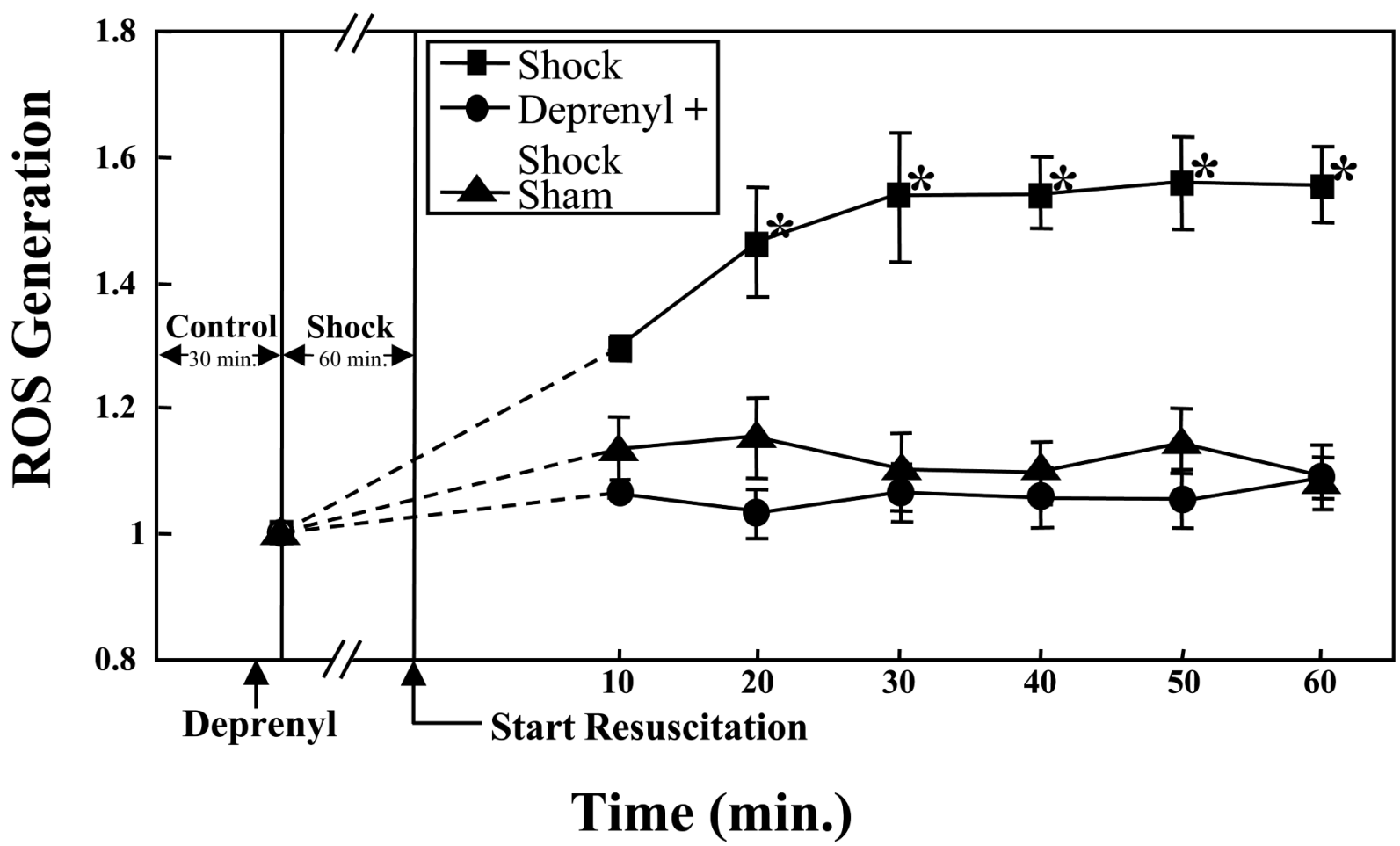

Figure 2.

A. (-)-Deprenyl decreases hemorrhagic shock induced mitochondrial ROS formation in rat mesentery post-capillary venules. The images of mesenteric post-capillary venules of preshock, hemorrhagic shock for 1 hour followed by 60 minutes of resuscitation $\left(\mathrm{T}_{60}\right)$ and (-)deprenyl treatment 10 minutes prior to hemorrhagic shock ((-)-deprenyl + Shock $\left.\mathrm{T}_{60}\right)$ are shown. Increased ROS formation is observed following hemorrhagic shock whereas (-)deprenyl treatment prevented the ROS formation. B. ROS formation is expressed as change in fluorescent intensity. Hemorrhagic shock induces ROS formation compared with sham-control group. (-)-Deprenyl treatment prevents HS-induced ROS formation compared with hemorrhagic shock group without (-)-deprenyl treatment. * $(p<0.05 ; \mathrm{n}=5)$. 


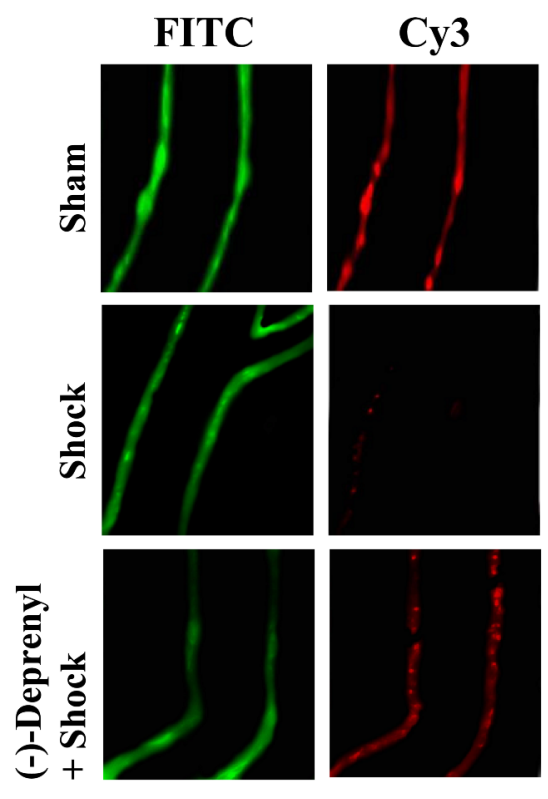

A

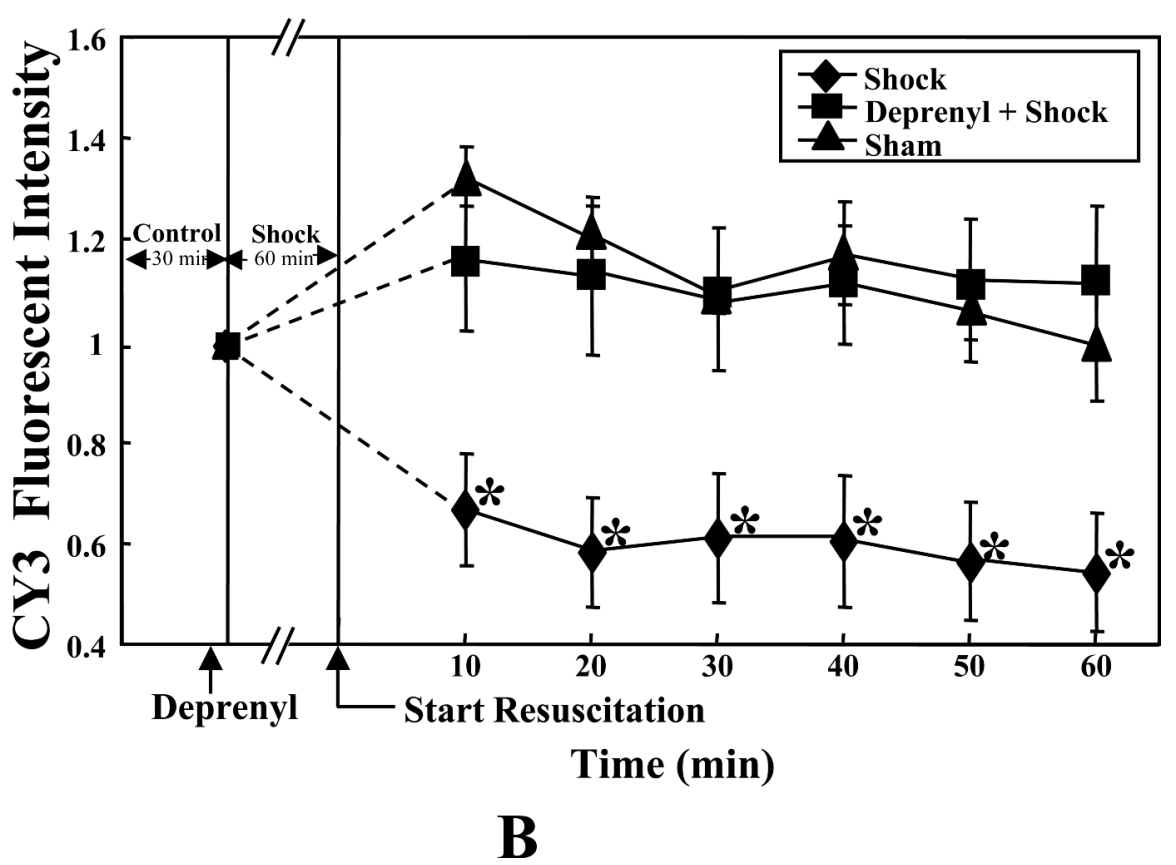

Figure 3.

A. (-)-Deprenyl prevents hemorrhagic shock induced decrease in mitochondrial transmembrane potential. In sham-control, the JC-1 fluoresced the mitochondria red (Cy3 filter) and the cytoplasm green (FITC filter), indicating intact mitochondria. Hemorrhagic shock for 1 hour followed by 60 minutes of resuscitation (Shock $\mathrm{T}_{60}$ ) induced the collapse of membrane potential allowing diffusion of the red fluorescence into the cytoplasm. (-)-Deprenyl treatment 10 minutes prior to hemorrhagic shock prevents the decrease in membrane potential evidenced by the presence of bright red fluorescence. B. Data represents the change in red fluorescence observed using a Cy 3 filter. Hemorrhagic shock for 1 hour followed by 60 minutes of resuscitation (Shock $\mathrm{T}_{60}$ ) induced the collapse of membrane potential allowing diffusion of the red (Cy3 filter) fluorescence into the cytoplasm. (-)-Deprenyl treatment 10 minutes prior to hemorrhagic shock prevents the decrease in membrane potential compared to the shock group $*(p<0.05 ; \mathrm{n}=5)$. 


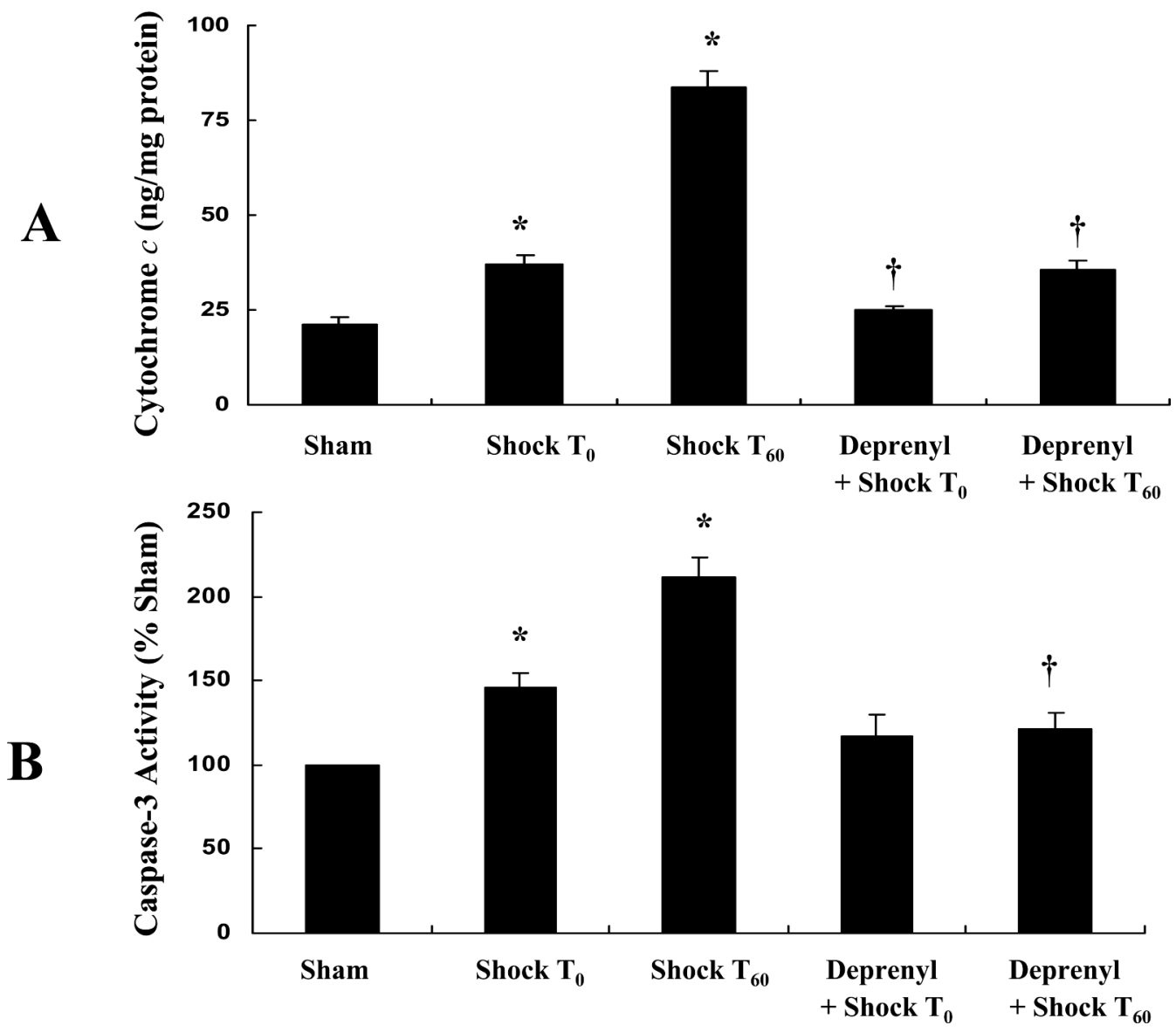

Figure 4.

A. (-)-Deprenyl prevents hemorrhagic shock induced release of cytochrome $c$ from mitochondria to the cytosol in rat mesenteric vasculature. Hemorrhagic shock for 1 hour followed by 0 or 60 minutes of resuscitation $\left(\mathrm{T}_{0}\right.$ or $\left.\mathrm{T}_{60}\right)$ significantly increases the cytosolic cytochrome $c$ levels. * $(p<0.05 ; \mathrm{n}=5)$. (-)-Deprenyl treatment 10 minutes prior to hemorrhagic shock $\left(\mathrm{T}_{0}\right.$ or $\left.\mathrm{T}_{60}\right)$ decreases cytochrome $c$ levels compared with hemorrhagic shock groups without (-)-deprenyl treatment. $\dagger(p<0.05 ; \mathrm{n}=5)$. B. (-)-Deprenyl prevents hemorrhagic shock induced activitation of caspase- 3 in rat mesenteric vasculature. Hemorrhagic shock for 1 hour followed by 0 or 60 minutes of resuscitation $\left(T_{0}\right.$ or $T_{60}$ ) significantly increased the caspase- 3 activity. $*(p<0.05 ; \mathrm{n}=5)$. (-)-Deprenyl treatment 10 minutes prior to hemorrhagic shock $\left(\mathrm{T}_{60}\right)$ significantly attenuates caspase- 3 activity compared with hemorrhagic shock $\left(\mathrm{T}_{60}\right)$ group without (-)-deprenyl treatment. $\dagger(p<0.05 ; \mathrm{n}=5)$. 


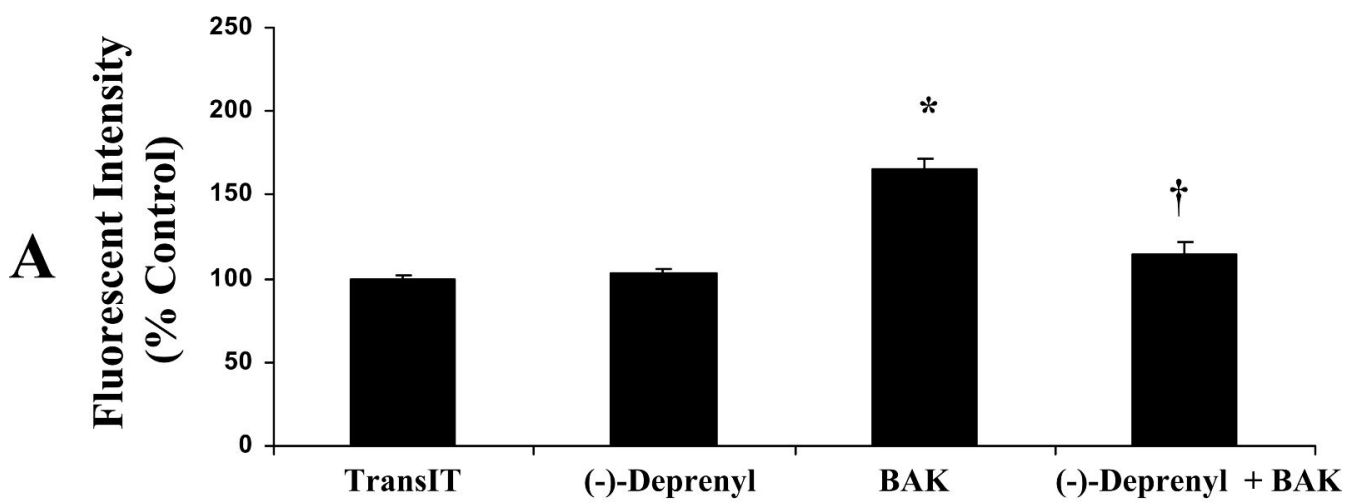

B

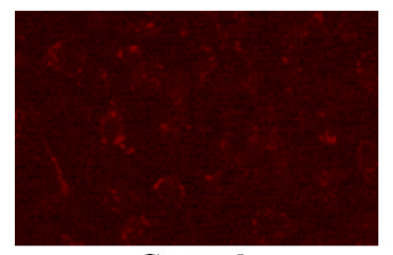

Control

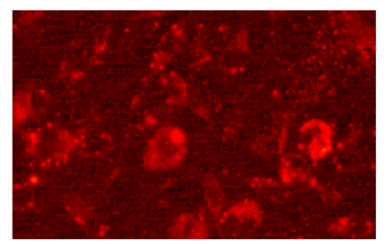

(-)-Deprenyl

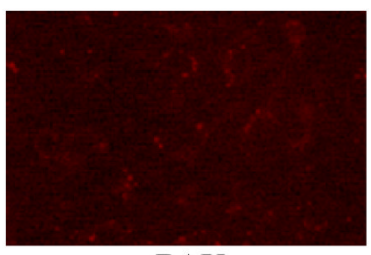

BAK

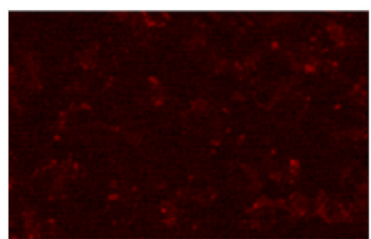

(-)-Deprenyl + BAK

Figure 5.

A. Effect of (-)-Deprenyl on BAK (BH3)-induced monolayer hyperpermeability. The FITCalbumin fluorescence intensity is significantly high in the BAK (BH3) peptide transfected group compared with the transfection reagent (TransIT) alone treated group $*(p<0.05 ; \mathrm{n}=5)$. Cells pretreated with (-)-deprenyl $(10 \mu \mathrm{M})$ show a decrease in BAK (BH3)-induced hyperpermeability compared with the cells transfected with BAK (BH3) and not pre-treated with (-)-deprenyl. $\dagger(p<0.05 ; \mathrm{n}=5)$. B. Effect of (-)-deprenyl on BAK (BH3)-induced ROS formation. In RLMEC, transfection of BAK (BH3) peptide shows increased red fluorescence indicating increased ROS formation compared with untreated control or (-)-deprenyl $(10 \mu \mathrm{M})$ alone treated cells. (-)-Deprenyl $(10 \mu \mathrm{M})$ pre-treated cells show less fluorescence. 


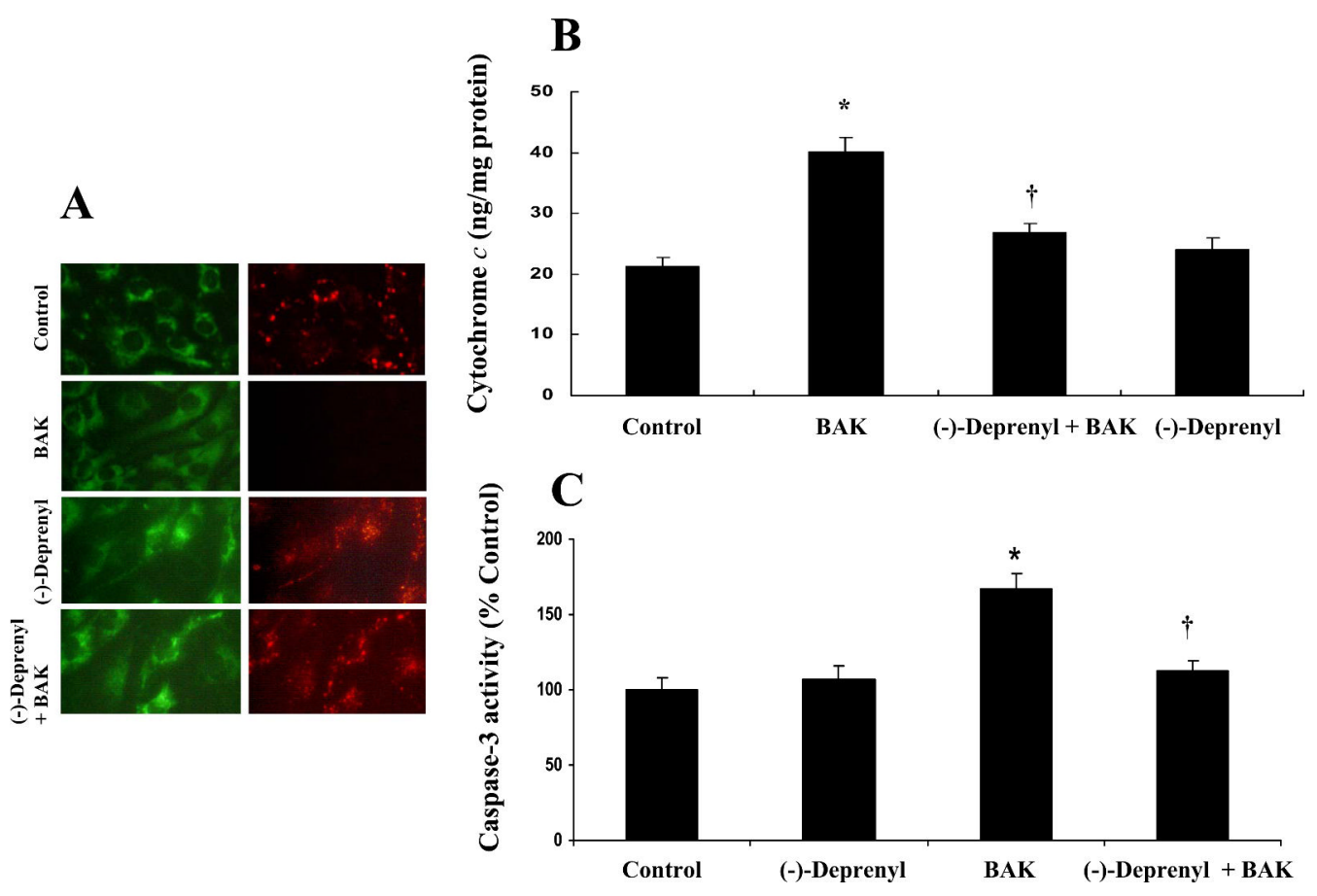

Figure 6.

A. Effect of (-)-deprenyl on BAK (BH3)-induced collapse of mitochondrial transmembrane potential. In untreated (control) cells and (-)-deprenyl $(10 \mu \mathrm{M})$ treated cells, JC-1 fluoresce red representing intact mitochondria. Following BAK $(\mathrm{BH} 3)$ transfection, there is a decrease in red (mitochondrial) fluorescence indicating the loss of mitochondrial transmembrane potential. BAK (BH3) transfected cells pre-treated with (-)-deprenyl shows red fluorescence indicating the recovery of mitochondrial membrane potential. B. Effect of (-)-Deprenyl on BAK (BH3)induced cytochrome $c$ release. In BAK (BH3) transfected cells, cytosolic levels of cytochrome $c$ is significantly higher in comparison to the control cells. $*(p<0.05 ; \mathrm{n}=5)$. The cytochrome $c$ levels show a significant decrease in BAK (BH3)-transfected cells pre-treated with (-)deprenyl $(10 \mu \mathrm{M})$ in comparison to BAK (BH3)-transfected cells without (-)-deprenyl pretreatment. $\dagger(p<0.05 ; \mathrm{n}=5)$. C. Effect of (-)-Deprenyl on BAK (BH3)-induced caspase-3 activity. In BAK (BH3) transfected cells, caspase-3 activity is significantly higher in comparison to the control group $*(p<0.05 ; \mathrm{n}=5)$. The caspase- 3 activity shows a significant decrease in BAK (BH3)-transfected cells pre-treated with (-)-deprenyl $(10 \mu \mathrm{M})$ in comparison to BAK (BH3)-transfected cells without (-)-deprenyl pre-treatment. $\dagger(p<0.05 ; \mathrm{n}=5)$. 